\title{
Dynamic analysis of a diffusing particle in a trapping potential
}

\author{
Moshe Lindner, ${ }^{1}$ Guy Nir, ${ }^{1}$ Anat Vivante, ${ }^{1}$ Ian T. Young, ${ }^{2}$ and Yuval Garini ${ }^{1}$ \\ ${ }^{1}$ Department of Physics, Institute of Nanotechnology, Bar Ilan University, Ramat Gan 52900, Israel \\ ${ }^{2}$ Department of Imaging Science and Technology, Delft University of Technology, Lorentzweg 1, CJ Delft 2628, The Netherlands
}

(Received 18 July 2012; published 25 February 2013)

\begin{abstract}
The dynamics of a diffusing particle in a potential field is ubiquitous in physics, and it plays a pivotal role in single-molecule studies. We present a formalism for analyzing the dynamics of diffusing particles in harmonic potentials at low Reynolds numbers using the time evolution of the particle probability distribution function. We demonstrate the power of the formalism by simulation and by measuring and analyzing a nanobead tethered to a single DNA molecule. It allows one to simultaneously extract all the parameters that describe the system, namely, the diffusion coefficient and the restoring-force constant.
\end{abstract}

DOI: 10.1103/PhysRevE.87.022716

PACS number(s): 87.80.Nj, 82.37.-j, 87.14.gk, 87.15.H-

\section{INTRODUCTION}

Diffusing particles under potential fields are of great interest in a broad range of physical systems, and the dynamics of such particles is specifically relevant in soft condensed matter physics and biophysics. Moreover, unprecedented biophysical information is provided through single-molecule methods that involve the dynamics of particles in trapping potentials. These include optical [1] and magnetic tweezers [2,3], atomic force microscopy [4], and tethered particle motion (TPM) [5]. Although the properties of diffusing particles in potential fields have gained much attention, the complete dynamics of these systems have not yet been fully explored. Treating the detailed dynamics of these systems provides both a better understanding of their properties and an improved capability for related experimental methods.

\section{THEORETICAL BACKGROUND}

For studying the detailed dynamic properties of a diffusing particle in a harmonic potential, we start by calculating its probability $P$ to be found at position $r$ and time $t$, given its initial position and time $r_{0}, t_{0}: P\left(r, t \mid r_{0}, t_{0}\right)$, see Fig. 1 . This can be calculated by using Smoluchowski's formalism [6], a special case of the Fokker-Planck equation that describes the dynamics of a diffusing particle in a stationary potential field. The Smoluchowski equation is derived for the case of low Reynolds numbers and, therefore, neglects the inertia term of the Langevin equation, which is the overdamped case. This solution is fully adequate for the experimental methods listed above for biophysical systems. In its simplest form, this system (and equation) has only two free parameters, the diffusion coefficient of the free particle $D$ and the restoring-force constant $K$. It was solved long ago [7,8], but its solution, that we further develop here, was overlooked. Moreover, previous treatments concentrated on the average displacement of the probability $P$, whereas, here, we show that the time evolution of both the average as well as the width of $P$ provides a better understanding of the dynamics and that its adaptation can improve the capability and effectiveness of single-molecule methods.

More specifically, the dynamic analysis can be used to extract the restoring force constant $K$ and the particle's diffusion coefficient $D$ simultaneously by time tracking the particle. Inversely, the method can be used to calculate the diffusion coefficient of particles, even if they can only be detected while they are trapped (e.g., by tethering them). Finally, the developed formalism intrinsically allows one to distinguish different time-dependent dynamic regimes: the fast dynamics that depend mainly on $D$, the intermediate dynamics that depend on both $D$ and $K$, and the slow dynamics that depend only on $K$.

The time evolution of the distribution probability $P$ of a diffusing particle at position $\mathbf{r}$ and time $t$, given its initial position $\mathbf{r}_{0}$ at time $t_{0}$ in a potential field, is given by the Smoluchowski equation,

$$
\frac{\partial}{\partial t} P\left(\mathbf{r}, t \mid \mathbf{r}_{0}, t_{0}\right)=\nabla \cdot D[\nabla-\beta \mathbf{F}(\mathbf{r})] P\left(\mathbf{r}, t \mid \mathbf{r}_{0}, t_{0}\right),
$$

where $D$ is the diffusion coefficient, $\beta=1 / k_{b} T$, and $\mathbf{F}(\mathbf{r})$ is the force applied on the particle. This equation is developed by using Einstein relations for the diffusion coefficient, drag coefficient $\zeta$, and thermal energy $D \zeta=k_{b} T$. In the case of one-dimensional diffusion in a harmonic potential, $U(x)=$ $K x^{2} / 2$, and assuming that the diffusion coefficient is an isotropic scalar, the equation can be written as

$$
\frac{\partial}{\partial t} P\left(x, t \mid x_{0}, t_{0}\right)=D\left(\frac{\partial^{2}}{\partial x^{2}}+\beta K \frac{\partial}{\partial x} x\right) P\left(x, t \mid x_{0}, t_{0}\right) .
$$

Equation (2) has an analytical solution [6,9],

$$
\begin{aligned}
& P\left(x, t \mid x_{0}, t_{0}\right) \\
& \quad=\sqrt{\frac{K}{2 \pi k_{b} T S\left(t, t_{0}\right)}} \exp \left(-\frac{\left(x-x_{0} e^{-2\left(t-t_{0}\right) / \tau}\right)^{2}}{2 k_{b} T S\left(t, t_{0}\right) / K}\right),
\end{aligned}
$$

where $S\left(t, t_{0}\right)=1-\exp \left[-4\left(t-t_{0}\right) / \tau\right]$ and $\tau=2 k_{b} T /$ $(K D)$. The solution, which is Gaussian, depends on the parameters $x_{0}$ and $\Delta t \equiv t-t_{0}$, and its time evolution is characterized by a time-dependent average and a width which is proportional to $S\left(t, t_{0}\right)$.

When a particle time track is measured, the Gaussian center and width can be used for extracting both $K$ and $D$, although it is mainly the Gaussian width that is sensitive to each of these two parameters independently. The Gaussian central position depends almost exclusively on $\tau$, which is a function of $K D$ and does not allow extraction of these parameters separately. As $\Delta t \rightarrow 0$, Eq. (3) approaches the Dirac $\delta$ function at $x_{0}: P$ $\left(x, \Delta t \rightarrow 0 \mid x_{0}\right)=\delta\left(x-x_{0}\right)$, and as $\Delta t \rightarrow \infty$, the solution 


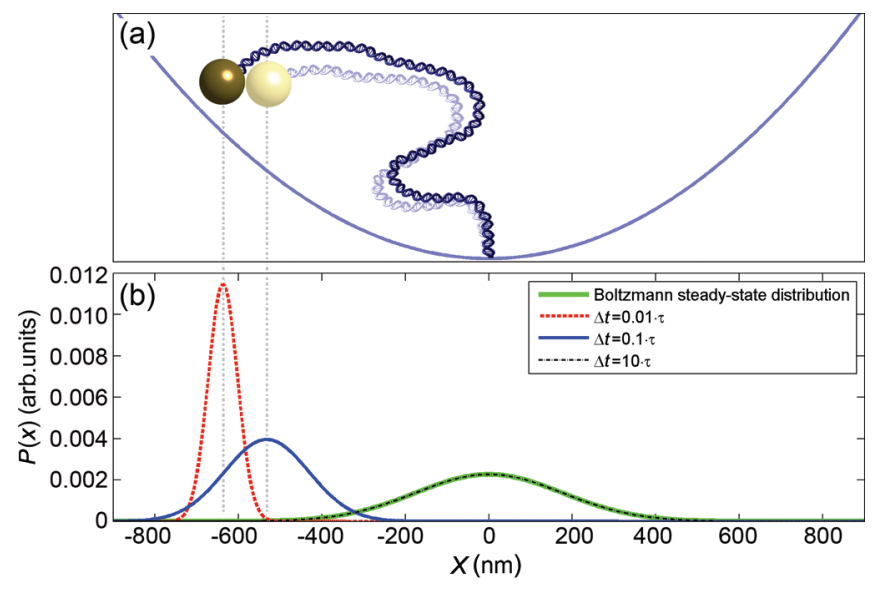

FIG. 1. (Color online) (a) Pictorial description of a single particle diffusing in a harmonic potential applied by the DNA. Two different conformations are shown. (b) $P\left(x, \Delta t \mid x_{0}\right)$ for $x_{0}=-650 \mathrm{~nm}$. Dashed red line: for $\Delta t=0.01 \tau$; solid blue line: for $\Delta t=0.1 \tau$; and dotted black line: for $\Delta t=10 \tau$. Solid green line: the steady-state (Boltzmann) distribution.

converges to the equilibrium Boltzmann distribution (Fig. 1),

$$
P(x)=\sqrt{K / 2 \pi k_{b} T} \exp \left(-K x^{2} / 2 k_{b} T\right) .
$$

For a general $\Delta t$, the solution of the Smoluchowski equation [Eq. (3)] has a Gaussian width of $\sigma^{2}=k_{b} T S / K$ that broadens with $\Delta t$, finally reaching a steady-state value [Eq. (4)]. The center of the distribution function depends on the initial position $x_{0}$ and converges exponentially as a function of time to $x=0$ with a relaxation time constant $\tau$. Actually, $\tau$ is identical to the correlation time that is found by calculating the time correlation of the position coordinate $\langle x(t) x(0)\rangle=k_{b} T K^{-1} \exp (-2 t / \tau)$ [6]. The relaxation time $\tau$ is also directly related to the "corner frequency" that is found in the frequency-response curve (power spectrum) commonly used for analyzing optical-tweezers experiments $f_{c}=(\pi \tau)^{-1}$ $[10,11]$. Note that, from the power spectrum, it is possible to extract only the corner frequency $f_{c}$, which is a function of $K D$, and these two parameters cannot be extracted separately as in our formalism.

The center of the Gaussian at a given (fixed) $\Delta t$ linearly depends on $x_{0}$ as can be seen in the exponent of Eq. (3): $x\left(t_{0}+\Delta t\right)=x\left(t_{0}\right) \exp (-2 \Delta t / \tau)$. Figures 2(a) and 2(b) show the joint probability distribution $P(x(t), x(t+\Delta t))$ for any given pair of $x(t)$ and $x(t+\Delta t)$ assuming a uniform distribution for $x(t)$ for a fixed time interval $\Delta t=0.01 \tau$ and $\Delta t=0.5 \tau$. The probability is higher where the color is brighter. Nevertheless, in an actual potential field, the probability of observing the particle at an initial position $x_{0}$ is not uniform but, instead, follows the steady-state Boltzmann distribution [Eq. (4)]. Taking this into consideration gives a different joint distribution as shown in Figs. 2(c) and 2(d). The dashed lines in Fig. 2 describe the linear fit to the distribution (the center of the Gaussians as explained above).

In order to find the width $\sigma^{2}$ and the slope of the joint probability, we plot $x(t+\Delta t)$ vs $x(t)$ for each measured point along the time series. Note that the two-dimensional histogram of this plot is the joint probability as shown for the theoretical solution in Figs. 2(c) and 2(d) and experimentally in Figs. 4(a)
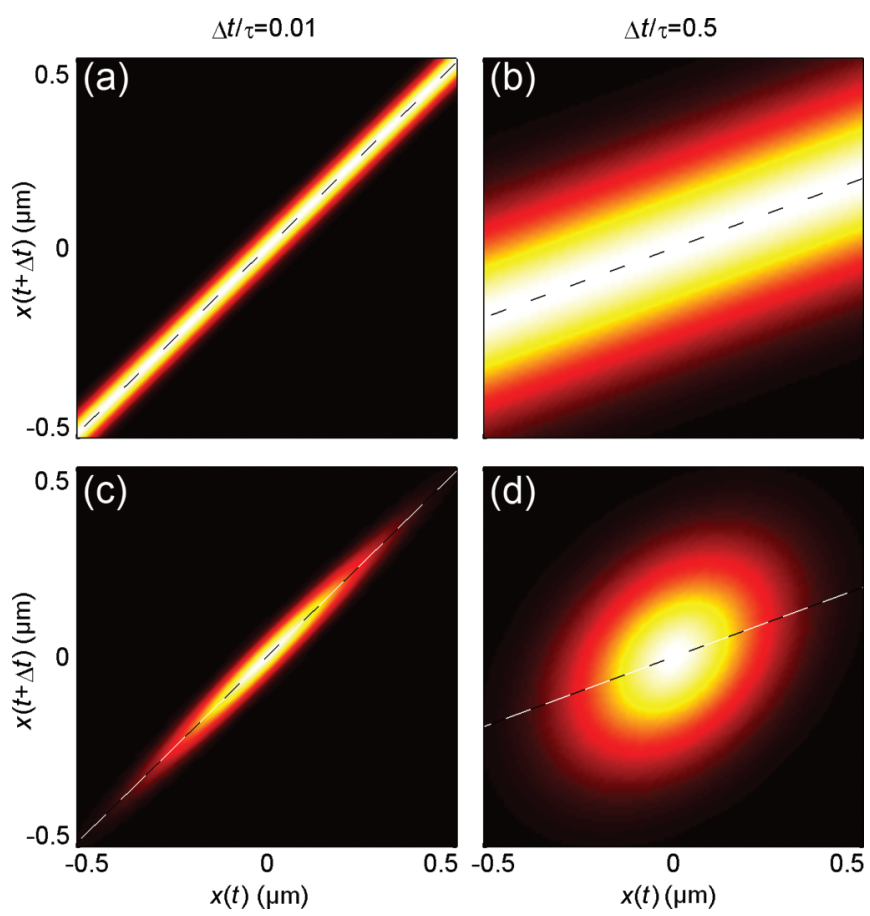

FIG. 2. (Color online) Theoretical joint probability distribution $P(x(t), x(t+\Delta t))$ for two different time intervals: left: $\Delta t=0.01 \tau$ and right: $\Delta t=0.5 \tau$. The brighter the color, the higher the probability. (a) and (b) Assuming that $x(t)$ is uniformly distributed. (c) and (d) Taking into account that the initial position $x(t)$ is distributed according to a Boltzmann distribution. The dashed lines represent the linear fit.

and 4(b). The slope is found by fitting this plot to a linear function. To extract the width $\sigma^{2}$, we subtract the linear curve that was fitted from the plot, project all the points on the vertical $x(t+\Delta t)$ axis, and obtain a set of points centered around zero. These points represent the distribution of distances of the particle in a given time interval $\Delta t$, which is independent of its previous position. $\sigma^{2}$ is now found by fitting a Gaussian to the distribution. An illustration of the process is available in Supplemental Material [12].

The slope shown for two different time intervals in Fig. 2 can be plotted as a function of any time interval $\Delta t$. When an experiment is performed, a certain fixed time interval $\Delta T$ is determined. Normally, the shortest time interval is preferred, but this is limited by the camera or detector and by the brightness of the detected particle. Based on that, different time intervals $\Delta t$ can be used: $\Delta t=\Delta T, 2 \Delta T, \ldots, n \Delta T$, and particle displacements for different time intervals can be calculated $x(t+\Delta t)-x(t)$. Figure 3(a) shows the slope as a function of the sample frequency $(f=1 / \Delta t)$ for both theory and simulation; details of the simulation are described later.

These results emphasize the existence of three different frequency domains for the dynamics of a diffusing particle in a force field. At long time intervals $\Delta t>\tau$ (low frequencies), the spatial dynamic correlation is lost, $\langle x(\Delta t) x(0)\rangle \approx 0$, the Boltzmann steady-state distribution is measured [Eq. (4)], and the slope mentioned above approaches zero. For short time intervals $\Delta t<\tau / 10$ (high frequencies), the effect of the restoring force on the particle displacement is negligible relative to the diffusion effect, and the slope approaches the 


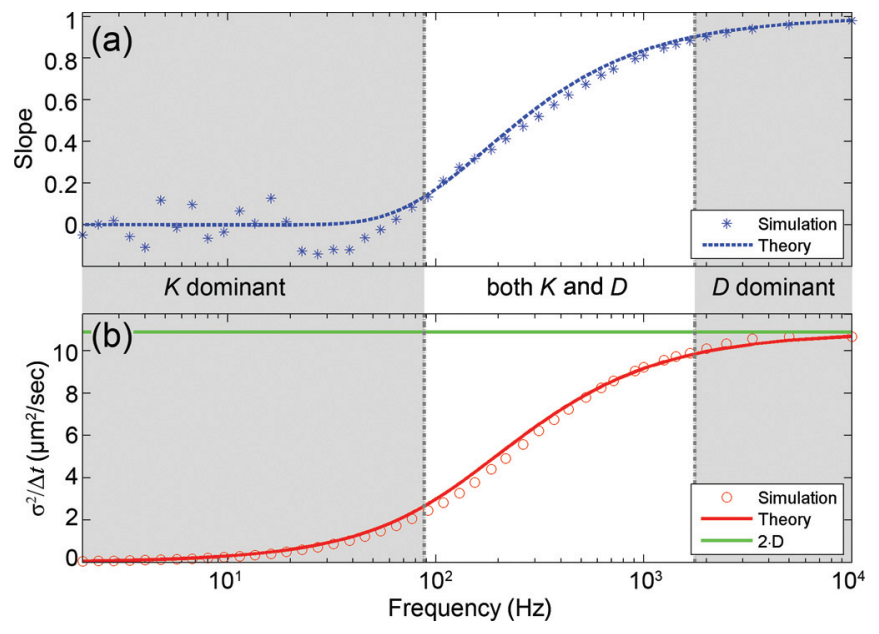

FIG. 3. (Color online) (a) Dashed blue line: the theoretical slope of the joint distribution $P(x(t), x(t+\Delta t))$ for different sample frequencies and asterisks: the simulation results. Three different frequency domains that correspond to different time-interval regimes are observed. At long time intervals (low frequencies), there is no correlation between the positions $\langle x(t) x(t+\Delta t)\rangle \approx 0$, and the steady-state distribution dominates. For short time intervals (high frequencies), the influence of the force field is negligible, and the diffusion dominates. In the intermediate regime, the force field, as well as the diffusion, affect the dynamics. (b) Solid red line: the theoretical Gaussian width as a function of the sampling frequency shown as $\sigma^{2} / \Delta t$ vs $(\Delta t)^{-1}$. Circles: simulation results. At high frequencies, the Gaussian width becomes identical to that of a one-dimensional freely diffusing particle, shown as a solid green line: $\sigma^{2} / \Delta t=2 D$.

value 1. The Gaussian width in this regime (derived from Taylor series expansion of $S$ ) is equal to $\sigma^{2} \approx 2 D \Delta t$ as expected for normal diffusion [13]. Finally, in the intermediate frequencies, the particle dynamics depends on both its free diffusion parameter $D$ as well as the DNA applied force parameter $K$.

This description, summarized in Fig. 3 for both (a) the slope and (b) the Gaussian width, clearly demonstrates the different frequency-response regimes of a diffusing particle in a harmonic-potential field and shows the advantages of using the dynamic measurement and the analysis according to Smoluchowski's equation. Notice that, in the low-frequencies regime, the slope suffers from errors due to statistical fluctuations, whereas, the width plot is insensitive to these fluctuations.

\section{EXPERIMENT AND SIMULATIONS}

To test the developed formalism, we have performed TPM simulations and experiments [5]. TPM belongs to a family of single-molecule methods that are intensively used for studying the fundamental properties of DNA, RNA, proteins, and their interactions. In this method, one end of a double-stranded DNA (or other polymer) is attached to the surface, and a detectable probe is attached to its other end. The DNA properties are normally extracted by analyzing the spatial distribution of the probe [14] as opposed to its actual dynamics that have been much less studied and are described hereunder.
The DNA can be modeled by using the wormlike chain polymer model, and its force-extension curve is described by [15]

$$
\frac{F l_{p}}{k_{b} T}=\frac{r}{L}+\frac{1}{4(1-r / L)^{2}}-\frac{1}{4},
$$

where $F$ is the force applied by a DNA molecule of contour length $L$ with an end-to-end extension $r$, a persistence length $l_{p}$, and a thermal energy $k_{b} T$.

In the low-force regime where $r<0.4 L$ and using a Taylor series expansion, Eq. (5) can be approximated as a linear force $F=K r$ with constant $K$, which results from pure entropic considerations [16],

$$
K=\frac{3 k_{b} T}{2 L l_{p}} .
$$

Few aspects of DNA dynamics have been treated before, such as the DNA dynamics in a strong stretching regime where $r \sim L$ [17], the DNA dynamics in response to stretching forces [18], or the dynamics of a tethered particle in the case of DNA looping [19,20]. Qian and Elson [21] used Smoluchowski's equation but eventually took into account only the steady-state solution and the autocorrelation function. Previous papers used different analysis methods including a time averaging approach [5], a steady-state distribution analysis [22,23], and the statistics of Markovian processes for measuring the rate of loop formation due to protein action [19,20]. The full dynamics as we described above, however, have not been considered.

The dynamic simulation is based on the code published by Beausang et al. [24]. In each time step of the simulation, the particle moves as a result of both the harmonic force and the diffusion. The parameters used relate to the experiment described below with $L=925 \mathrm{~nm}$ and $l_{p}=50 \mathrm{~nm}$, which gives a spring constant of $K=0.133 \mathrm{pN} / \mu \mathrm{m}$ at room temperature. The probe attached to the DNA is a bead with a diameter of $80 \mathrm{~nm}$, and its diffusion coefficient in water is $D=5.5 \mu \mathrm{m}^{2} / \mathrm{s}$. The simulation results are analyzed by finding the slope and width of the joint probability distribution $P(x(t), x(t+\Delta t))$ for different time intervals $\Delta t$ as explained above. The slope and Gaussian width are shown in Fig. 3. By performing simulations $(N=1000)$, we obtained the values of $K=0.135 \pm 0.015 \mathrm{pN} / \mu \mathrm{m}$ and $D=5.5 \pm 0.18 \mu \mathrm{m}^{2} / \mathrm{s}$ from the width fit [Fig. 3(b)]. These parameters agree very well with the theoretical derivation from the Smoluchowski equation given above.

The TPM experimental system details are described elsewhere $[25,26]$. The particle positions are tracked using a microscope and a CCD camera. Different experimental aspects have to be taken into account including the size of the particle relative to the DNA length [27] and the time needed for the DNA to "search" its conformational space [28].

We used a 2722 bp $(L=925 \mathrm{~nm}) \lambda$-DNA attached to the surface through digoxigenin (DIG)-anti-DIG conjugation and an $80 \mathrm{~nm}$ diameter gold bead attached to the DNA through a biotin-anti-biotin conjugation. A dark-field setup was used for the illumination and detection based on a $50 \times / 0.8$ numerical aperture dark-field objective lens (Olympus) and an electron-multiplied CCD camera (Andor DU-885). Image processing was performed using MATLAB and the DIPimage 

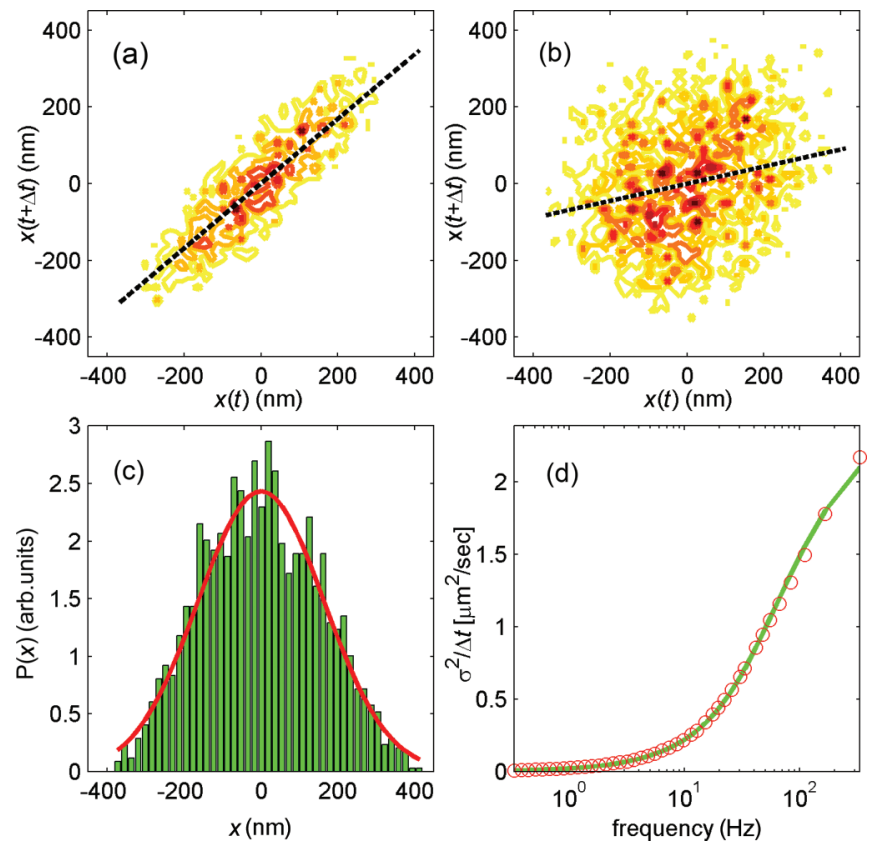

FIG. 4. (Color online) Experimental results. (a) $x(t+\Delta t)$ vs $x(t)$ for $\Delta t=3 \mathrm{~ms}$. (b) For $\Delta t=30 \mathrm{~ms}$, notice the changes in both slope and width. Dashed line: fit of a linear curve for the slope. (c) Bars: steady-state distribution. Solid red line: fit to a Gaussian. (d) Circles: experimental results for the width of the distribution divided by the time interval as a function of sample frequency. Solid line: fit to the theoretical function.

library (see Ref. [29]), and the fits were carried out using the non-linear-least-squares algorithm of MATLAB. In each experiment, a time series of 3000 frames was taken at a high frame rate of $\sim 300-500 \mathrm{~Hz}$. After preliminary analysis, the data were filtered for beads with symmetric motion in the $x-y$ plane that were attached to a single DNA molecule.

\section{RESULTS AND DISCUSSION}

Figures 4(a) and 4(b) show the joint distribution $P(x(t), x(t+\Delta t))$ for two different time intervals where the different slopes (dashed line) and widths are clearly noticeable. Figure 4(c) shows the steady-state distribution and fit, whereas, Fig. 4(d) shows the width as a function of the sampling frequency. As one can see, the results agree well with the theory (solid line). We extracted the force constant by fitting the steady-state distribution to the expected Boltzmann distribution [Eq. (4), shown in Fig. 4(c)], which gave $K=0.17 \pm 0.02 \mathrm{pN} / \mu \mathrm{m}$. This is a possible method for TPM, but it is much more difficult and is rarely used for the calibration of optical and magnetic tweezers, which are more complex systems as explained below. It is also possible to fit the slope vs $f$ as shown in Fig. 3(a), but as explained above, this method only extracts $\tau$, which is a function of $K D$, and for finding $K$ itself, a priori knowledge of $D$ is required [30]. This step of using a supposedly known diffusion coefficient may result in a large error.

In contrast, the frequency dependence of the Gaussian width [Fig. 4(d)] can be used to simultaneously extract both $K$ and $D$. The values extracted from the width function based on the $x$-axis data are as follows: $K=0.22 \pm 0.04 \mathrm{pN} / \mu \mathrm{m}$, which is close to the steady-state value, and $D=1.81 \pm$ $0.82 \mu \mathrm{m}^{2} / \mathrm{s}$. We note that such an analysis is possible from neither the steady-state distribution nor the derivation of the corner frequency, which is often used for extracting the force constant in tethered experiments. By using the steady-state distribution, it is possible to find $K$ [as in Fig. 4(c)], and then, by extracting $\tau$ from the slope (or the corner frequency that was described above), one can deduce the diffusion coefficient $D$. Nevertheless, this method is problematic, and it is rarely used since the steady-state analysis is vulnerable to low-frequency noise and drift. This noise results from mechanical vibrations and drift, and it is much slower than the typical frequency that characterizes the system $(f \sim 1 / \tau)$. The dynamic analysis method described here depends on the entire frequency spectrum, and therefore, it is much less sensitive to drift and noise.

Interestingly, we realized that the beads we measured were consistently bigger than what we a priori thought (by extracting the size from the diffusion coefficient). We attribute this to the fact that the beads that have been measured in the experiment are the brighter ones, which are also the larger ones, and their actual diffusion coefficient is, therefore, smaller. Another possible reason for the deviation of $D$ from its expected value, based on Stokes-Einstein calculation, is due to the complexity that may occur as a result of hydrodynamic effects near the surface and the bead-DNA molecule interaction.

\section{CONCLUSIONS}

These results verify the validity of the dynamic method based on Smoluchowski's formalism and demonstrate its advantage for extracting both $K$ and $D$. It can be used in most of the single-molecule experiments as long as the sampling frequency is high enough. It does not require modification of the experimental setup as needed in some optical-tweezers calibration methods [31]. Our dynamic method can also be useful when the diffusion is more complex [32], and it can be extended to describe the dynamics of particles exhibiting anomalous diffusion in potential fields. This may be important for understanding the structure and function in a crowded environment, such as the chromatin in the nucleus. We recently found that the dynamics in the nucleus is transient anomalous, a complex type of diffusion [33,34], but more work is required for these cases. The method can also be used for generalized variables, such as the angular parameter in torque-determination experiments [35]. We mention in passing that, even if the potential function is not harmonic $U(x) \sim x^{2}$, the linear restoring-force approximation can still be applied as long as the particle position is near the minimum of the potential. If this is not the case and the linear approximation is not adequate, one can find the solution of Smoluchowsik's equation for the nonharmonic potential (either analytically or numerically) and can use it to extract the force.

To summarize, we have used the Smoluchowski equation and its solution for developing a formalism to analyze the dynamics of a diffusing particle in a harmonic potential and have shown its advantages for simultaneously extracting the 
restoring-force constant and diffusion coefficient. We have shown the excellent fit of the simulated data with the theory and have demonstrated the applicability of our methodology in the TPM experiment. The dynamic analysis that we present can be important for applications, such as calibration of the force applied by an optical or magnetic tweezers and can significantly improve the accuracy of the extracted results. This formalism can also be extended to describe nonharmonic potentials where an adequate solution of the Smoluchowski equation can be used as a reference for fitting the experimental data. Furthermore, the formalism can be extended to describe the dynamics of particles exhibiting anomalous diffusion in potential fields, which may be of great importance for understanding fundamental mechanisms, such as the structure and function of molecules in a crowded environment.

\section{ACKNOWLEDGMENTS}

This work was supported, in part, by the Israel Science Foundation, Grants No. 985/08, No. 1729/08, and No. 1793/07 and by the Wolfson Foundation Grant No. 2008. We thank Professor R. Metzler for critical reading of the manuscript.
[1] M. D. Wang, H. Yin, R. Landick, J. Gelles, and S. M. Block, Biophys. J. 72, 1335 (1997).

[2] J. van Noort, S. Verbrugge, N. Goosen, C. Dekker, and R. T. Dame, Proc. Natl. Acad. Sci. USA 101, 6969 (2004).

[3] S. B. Smith, L. Finzi, and C. Bustamante, Science 258, 1122 (1992).

[4] C. Bustamante, S. B. Smith, J. Liphardt, and D. Smith, Curr. Opin. Struct. Biol. 10, 279 (2000).

[5] D. A. Schafer, J. Gelles, M. P. Sheetz, and R. Landick, Nature (London) 352, 444 (1991).

[6] M. Doi and S. F. Edwards, The Theory of Polymer Dynamics (Oxford University Press, Oxford, 1988).

[7] S. Chandrasekhar, Rev. Mod. Phys 15, 1 (1943).

[8] H. Risken, The Fokker-Planck Equation (Springer, Berlin, 1989).

[9] K. Schulten and I. Kosztin, Stochastic Methods in Biological Physics (Cambridge University Press, Cambridge, UK) (in press).

[10] K. Svoboda and S. M. Block, Annu. Rev. Biophys. Biomol. Struct. 23, 247 (1994).

[11] G. Volpe, R. Quidant, G. Badenes, and D. Petrov, Phys. Rev. Lett. 96, 238101 (2006).

[12] See Supplemental Material at http://link.aps.org/supplemental/ 10.1103/PhysRevE.87.022716 for an animation illustrating the width calculation process.

[13] S. K. Sainis, V. Germain, and E. R. Dufresne, Phys. Rev. Lett. 99, 018303 (2007)

[14] H. Jensenius and G. Zocchi, Phys. Rev. Lett. 79, 5030 (1997).

[15] J. F. Marko and E. D. Siggia, Macromolecules 28, 8759 (1995).

[16] M. Rubinstein and R. H. Colby, Polymer Physics (Oxford University Press, Oxford, 2003), p. 440.

[17] Y. Bohbot-Raviv, W. Z. Zhao, M. Feingold, C. H. Wiggins, and R. Granek, Phys. Rev. Lett. 92, 098101 (2004).

[18] A. Crut, D. A. Koster, R. Seidel, C. H. Wiggins, and N. H. Dekker, Proc. Natl. Acad. Sci. USA 104, 11957 (2007).
[19] J. F. Beausang, C. Zurla, C. Manzo, D. Dunlap, L. Finzi, and P. C. Nelson, Biophys. J. 92, L64 (2007).

[20] J. F. Beausang and P. C. Nelson, Phys. Biol. 4, 205 (2007).

[21] H. Qian and E. L. Elson, Biophys. J 76, 1598 (1999).

[22] M. Lindner, G. Nir, S. Medalion, H. R. C. Dietrich, Y. Rabin, and Y. Garini, Phys. Rev. E 83, 011916 (2011).

[23] S. Brinkers, H. R. C. Dietrich, F. H. de Groote, I. T. Young, and B. Rieger, J. Chem. Phys. 130, 215105 (2009).

[24] J. F. Beausang, C. Zurla, L. Finzi, L. Sullivan, and P. C. Nelson, Am. J. Phys. 75, 520 (2007).

[25] M. Lindner, G. Nir, H. R. C. Dietrich, Ian T. Young, E. Tauber, I. Bronshtein, L. Altman, and Y. Garini, Isr. J. Chem. 49, 283 (2009).

[26] G. Nir, M. Lindner, H. R. C. Dietrich, O. Girshevitz, C. E. Vorgias, and Y. Garini, Biophys. J. 100, 784 (2011).

[27] D. E. Segall, P. C. Nelson, and R. Phillips, Phys. Rev. Lett. 96, 088306 (2006).

[28] M. Manghi, C. Tardin, J. Baglio, P. Rousseau, L. Salomé, and N. Destainville, Phys. Biol. 7, 046003 (2010).

[29] www.diplib.org.

[30] J.-C. Meiners and S. R. Quake, Phys. Rev. Lett. 82, 2211 (1999).

[31] S. F. Tolić-Nørrelykke, E. Schäffer, J. Howard, F. S. Pavone, F. Jülicher, and H. Flyvbjerg, Rev. Sci. Instrum. 77, 103101 (2006).

[32] J.-H. Jeon, V. Tejedor, S. Burov, E. Barkai, C. Selhuber-Unkel, K. Berg-Sørensen, L. Oddershede, and R. Metzler, Phys. Rev. Lett. 106, 048103 (2011).

[33] I. Bronstein, Y. Israel, E. Kepten, S. Mai, Y. Shav-Tal, E. Barkai, and Y. Garini, Phys. Rev. Lett. 103, 018102 (2009).

[34] E. Barkai, Y. Garini, and R. Metzler, Phys. Today 65(8), 29 (2012).

[35] F. Pedaci, Z. Huang, M. van Oene, and N. H. Dekker, Opt. Express 20, 3787 (2012). 